\title{
Fluorescent amino acid initiated de novo cyclic peptides for the label-free assessment of cell permeability
}

\author{
Yuteng Wu, ${ }^{\dagger[a, b]}$ M. Teresa Bertran, ${ }^{\dagger[b]}$ James Rowley, ${ }^{[a, b]}$ Ewen D. D. Calder, ${ }^{[a, b]}$ Dhira Joshi, ${ }^{[c]}$ and \\ Louise J. Walport ${ }^{*[a, b]}$
}
[a] Y. Wu, M. T. Bertran, J. Rowley, E. D. D. Calder, L. J. Walport
Protein-Protein Interaction Laboratory
The Francis Crick Institute
London NW1 1AT, UK
E-mail: Louise.walport@crick.ac.uk
[b] Y. Wu, J. Rowley, E. D. D. Calder, L. J. Walport
Department of Chemistry
Molecular Sciences Research Hub
Imperial College London
London W12 OBZ, UK
[b] D. Joshi
Peptide Chemistry
The Francis Crick Institute
London NW1 1AT, UK
† These authors contributed equally to this work.

Supporting information for this article is given via a link at the end of the document.

\begin{abstract}
The major obstacle in applying peptides to intracellular targets is their low inherent cell permeability. Standard approaches to attach a fluorophore (e.g. FITC, TAMRA) can change the physicochemical properties of the parent peptide and influence their ability to penetrate and localize in cells. We report a label-free strategy for evaluating the cell permeability of cyclic peptide leads. Fluorescent tryptophan analogues 4-cyanotryptophan (4CNW) and $\beta$-(1-azulenyl)L-alanine (AzAla) were incorporated into in vitro translated macrocyclic peptides by initiator reprogramming. We then demonstrate these efficient blue fluorescent emitters are good tools for monitoring peptide penetration into cells.
\end{abstract}

In recent years cyclic peptides have emerged as promising drug candidates that can combine therapeutic benefits from both small molecules and biologics. ${ }^{[1,2]}$ They show excellent selectivity and tight binding to their targets, and their ability to interact with shallow surfaces makes them suitable for tackling challenging surfaces such as those involved in Protein-Protein Interactions. ${ }^{[3]}$ High affinity de novo cyclic peptides can be efficiently identified from enormous encoded peptide libraries using e.g. Phage or mRNA display platforms $\left(10^{8}-10^{14}\right.$ members). In particular the Random nonstandard Peptides Integrated Discovery (RaPID) allows the identification of cyclic peptides containing a wide range of unnatural amino acids. ${ }^{[4]}$

One major challenge for the application of peptides to intracellular targets, however, is their low inherent cell permeability. Straightforward assays are therefore required to assess the permeability of promising leads, with fluorescence imaging being a popular approach. Typically, peptide leads are appended with a fluorophore (e.g. FITC, TAMRA, Rhodamine) to assess cell permeability. ${ }^{[5]}$ Whilst widely used, these strategies use the labelled peptide as a proxy for the untagged version of the molecule. This can result in misleading findings where the fluorophore changes the physicochemical properties of the parent peptide and influences their ability to penetrate and localize in cells. ${ }^{[6,7]}$ Use of minimally perturbing dyes, or label-free assays are therefore the gold standard for determining the cell permeability of cyclic peptide leads.

Recently, non-canonical amino acids 4-cyanotryptophan (4CNW) and $\beta$-(1-azulenyl)-L-alanine (AzAla) have been shown to be efficient blue fluorescent emitters. ${ }^{[8,9]}$ Benefiting from their close structural similarity with tryptophan, incorporation of such molecules into cyclic peptides is potentially less disruptive to overall structure and permeability properties. Inspired by this, we set out to explore the introduction of $4 \mathrm{CNW}$ and AzAla into cyclic peptides by ribosomal translation. We selected the flexible in vitro translation system (FIT), which uses Flexizymes (small flexible tRNA acylation ribozymes) to prepare nonproteinogenic acyl-tRNAs using chemically activated amino

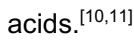

In a typical RaPID selection, the initiator is reprogrammed with an $\mathrm{N}$ chloroacetylated amino acid, that readily undergoes peptide cyclisation with a downstream cysteine after in vitro translation of the linear peptide. ${ }^{[11-13]}$ We designed compounds CIAc-4CNW-CME 7 and CIAc-AzAla-CME 8 to explore the inclusion of fluorescent amino acids into in vitro translated cyclic peptides via initiator reprogramming. The chloroacetyl (CIAc) group facilitates peptide cyclisation with a cysteine residue following translation, and the cyanomethyl ester (CME) acts as an efficient leaving group for flexizyme-mediated tRNA charging. Amino acids $\mathbf{3}$ and $\mathbf{4}$ were obtained by enzymatically assembling 4cyanoindole (1) or azulene (2) with serine using a tryptophan synthase $\beta$-subunit (TrpB) variant $\left(\mathrm{Tm} \mathrm{DD} 8^{*}\right)$ developed by Arnold and coworkers. ${ }^{[14]}$ Treatment of $\mathbf{3}$ and $\mathbf{4}$ with $\mathrm{N}$-(chloroacetoxy)succinimide gave intermediates $\mathbf{5}$ and $\mathbf{6}$ in moderate yields. Finally, esterification of the carboxylic acids with chloroacetonitrile installed the CME functionality of $\mathbf{7}$ and 8 (Scheme 1). 
a

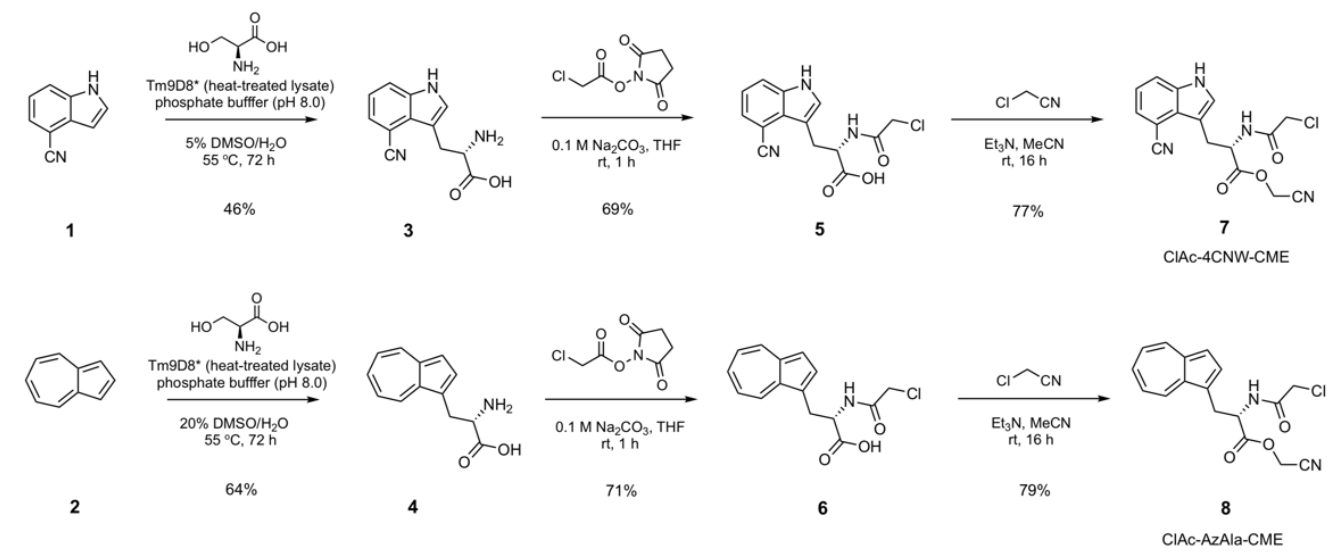

Scheme 1. Three-step preparation of CIAc-4CNW-CME 7 (a) and CIAc-AzAla-CME 8 (b).

With compounds $\mathbf{7}$ and $\mathbf{8}$ in hand, we set out to assess their tRNA acylation efficiency by using a tRNA analogue, the microhelix RNA (FAM-MiHx_23b) (Figure 1). The FAM label allows for visualisation through in gel fluorescence and loading yields were calculated by densitometric analysis of RNA bands. Initial studies were carried out with CIAC-4CNW-CME 5 at $\mathrm{pH}$ 7.5. We observed a gradual improvement in acylation yields (33-39\%) as reaction time increased from 2 to $16 \mathrm{~h}$. Altering the $\mathrm{pH}$ to 9 significantly improved $\mathrm{MiHx}$ charging, reaching $50 \%$ after $2 \mathrm{~h}$, but prolonging incubation time under these conditions did not further increase yields. A similar reactivity profile was observed for CIAc-AzAla-CME 6, with the higher $\mathrm{pH}$ giving the highest $\mathrm{MiHx}$ loading (50\%).
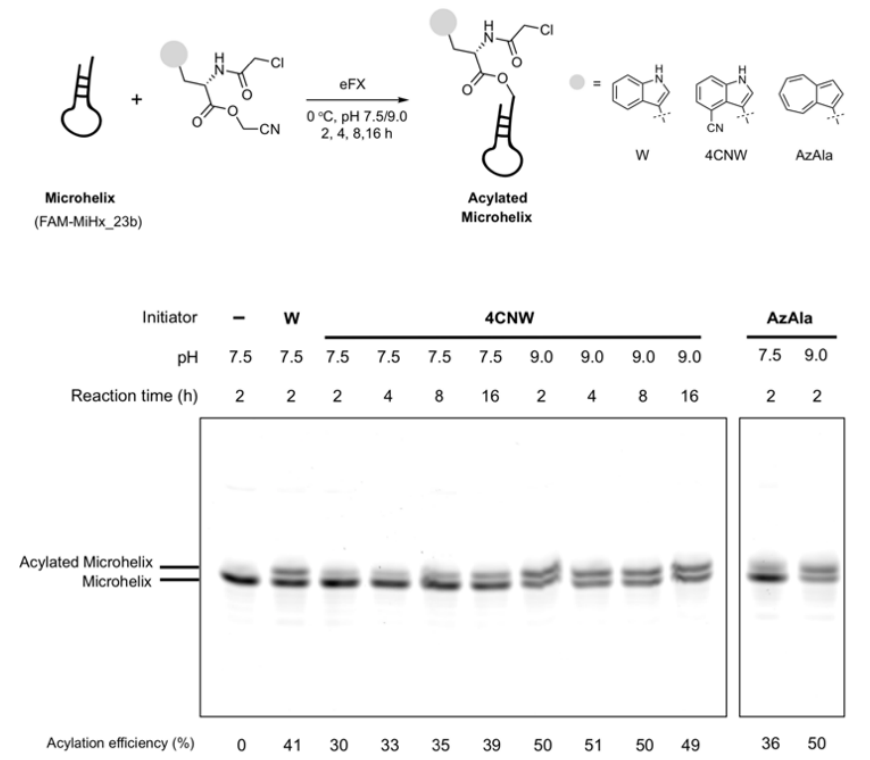

Figure 1. eFx-mediated aminoacylation of microhelix RNA (FAMMiHx_23b). Reaction mixtures were analyzed on a $20 \%$ polyacrylamide gel by detection of the FAM label. Acylation efficiencies were calculated by densitometric analysis of RNA bands. Substrates tested: CIAc-W-CME, CIAc4CNW-CME 7, CIAc-AzAla-CME 8.

Using the optimised conditions ( $\mathrm{pH}$ 9.0, $2 \mathrm{~h}$ ) from the MiHx loading assay, substrates 7,8 were charged onto initiator tRNA ${ }^{\text {fMet }}$ CAu through eFx mediated aminoacylation (Figure 2a). The resulting conjugates were then supplemented to a Met-free FIT system (PURExpress ${ }^{\mathrm{TM}}$, $\triangle$ aa, $\triangle$ tRNA, NEB). Synthesis of a model cyclic peptide P1 initiated with the fluorescent amino acids or $\mathrm{ClAc}-\mathrm{W}$ as a control, was monitored by MALDI-TOF mass spectrometry (MS). The translated cyclic peptide products were detected for both fluorescent initiators (Figure 2b). We noted a minor mass peak corresponding to the incorporation of methionine as the initiator was present for all translations (including the control with $\mathrm{Cl}-\mathrm{W}-\mathrm{CME}$ ), presumably arising from charged tRNA ${ }^{\text {fMet }}$ present in the commercial PURExpress kit.

We also investigated whether fluorescent amino acids 3,4 could be directly incorporated into a model linear sequence $\mathbf{P} 2$ by mischarging onto the tryptophan tRNA by tryptophanyl-tRNA synthetase. Amino acids 3, 4 were supplemented to a Trp-free FIT system. MALDI-TOF MS analysis showed successful expression of the 4-cyanotryptophan containing peptide $\mathbf{4 C N W - P 2}$, indicating good tolerance of $\mathbf{3}$ with tryptophanyl-tRNA synthetase to allow efficient tRNA charging and subsequent translation (Figure 2c). However, consistent with a previous report, ${ }^{[15]}$ attempts at incorporating AzAla $\mathbf{4}$ were unsuccessful, presumably due to failed recognition by tryptophanyltRNA synthetase owing to ring expansion and removal of the indole amine.

We then explored the fluorescent properties of the in vitro translated peptide containing $4 \mathrm{CNW}$. Translated reaction mixtures $(10 \mu \mathrm{L})$ were run in a Tricine-SDS PAGE gel and imaged with a trans-UV $302 \mathrm{~nm}$ excitation light source. In-gel fluorescence was observed for the translation sample with $4 \mathrm{CNW}$, but not with tryptophan (Figure 2d) confirming that $4 \mathrm{CNW}$ is a highly sensitive fluorophore that can be detected in low quantities, such as those present in a $10 \mu \mathrm{L}$ in vitro translation mixture. In-gel visualisation of AzAla $\left(\lambda_{\max }=276 \mathrm{~nm}\right)^{[15]}$ was not attempted due to a suitable excitation light source being unavailable. 


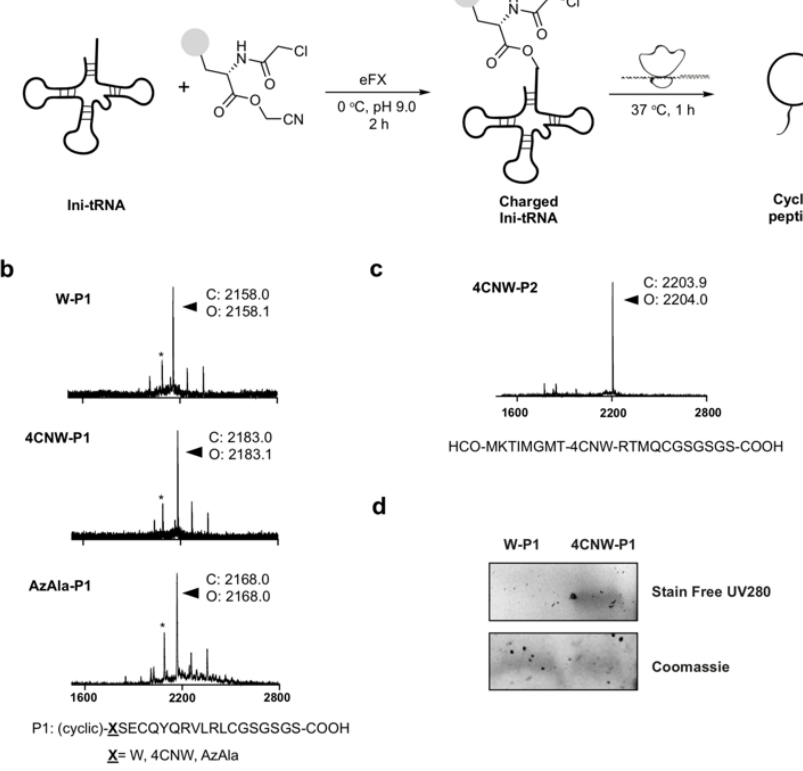

Figure 2. 4CNW/AzAla can be incorporated into a peptide by in vitro translation. a Charging of initiator tRNA followed by in vitro translation to give cyclic peptides. b MALDI-TOF MS spectra of translated cyclic peptides W-P1, 4CNW-P1, AzAla-P1 ( ${ }^{*}=$ HCO-MSECQYQRVLRLCGSGSGS-COOH). c MALDI-TOF MS spectrum of translated linear peptide 4CNW-P2. d Fluorescence visualization of translated 4CNW-P1 on a Tricine-SDS PAGE gel.

Finally, we assessed the potential of using either 4CNW or AzAla for biological imaging of peptides. Gai and co-workers have previously demonstrated $4 \mathrm{CNW}$ as a satisfactory fluorophore for imaging by coupling to an antimicrobial peptide. ${ }^{[9]}$ However, to the best of our knowledge, the use of AzAla in fluorescence microscopy has not yet been explored. To evaluate their suitability for tracking peptide penetration into cells, 4CNW/AzAla were appended onto the $\mathrm{N}$ terminus of a well-characterized cell penetrating peptide, TAT-NLS P3 (tryptophan was included as a negative control). Human bone osteosarcoma epithelial cells (U2OS) were incubated with peptides 4CNW-P3, AzAla-P3, W-P3 for 20 minutes at a concentration of 50 $\mu \mathrm{M}$ in OPTI-MEM. Cells were then washed once with OPTI-MEM and imaged on a Nikon Ti Eclipse inverted microscope with fluorescence excitation at $340 \mathrm{~nm}$. An ET460/50m single bandpass emission filter was used to image 4CNW-P3 and a ET395/25x single bandpass emission filter was used to image AzAla-P3. Our images show considerable intracellular fluorescence suggesting $4 \mathrm{CNW}$ and AzAla are suitable fluorophores for evaluating cell permeability of peptides when coupled with fluorescence microscopy (Figure 3a). We noted significant levels of cytotoxicity for all three TAT-NLS peptides over longer incubation periods (e.g. $2 \mathrm{~h}$ ), as measured by the release of lactate dehydrogenase (Figure S6). We envisage the cytotoxicity may be attributed to the combination of the TAT-NLS sequence and the additional $\mathrm{N}$-terminal appendage, such that the evaluation of cell viability must be considered when combining these fluorescent amino acids with novel peptide sequences.

To validate the use of $4 \mathrm{CNW}$ in studying the cell penetration of de novo cyclic peptides, we also substituted $4 \mathrm{CNW}$ for an internal tryptophan residue in a recently reported $\mathrm{K} 48 \mathrm{Ub}$ chain-binding cyclic peptide P4, identified using the RaPID system. ${ }^{[16]}$ To assess if $\mathbf{4 C N W}$. P4 was penetrating cells, U2OS cells were incubated with $50 \mu \mathrm{M}$ peptide for $24 \mathrm{~h}$. Cells were then imaged by fluorescence microscopy using the same conditions as described above. As shown in Figure 3b, appreciable intracellular fluorescence was observed suggesting efficient uptake of the cyclic peptide, consistent with data previously reported. ${ }^{[16]}$

Flow cytometry has been widely used to study internalization of fluorescently tagged peptides in cells. Therefore, we wanted to explore the use of $4 \mathrm{CNW}$ for monitoring cellular uptake of peptides by flow cytometry. U2OS cells were incubated with $50 \mu \mathrm{M}$ of $\mathbf{4 C N W}-\mathbf{P} 3$ or 4CNW-P4 at different time points and the fluorescence of a single cell solution was measured. After $4 \mathrm{~h}$ incubation with 4CNW-P4 we could detect a small percentage of positive blue fluorescent cells and this percentage was slightly increased at longer incubation times (Figure 3c, Figure S7). Incubation of cells with 4CNW-P3 for 20 minutes was sufficient for initiating the detection of positive cells. These data indicate that flow cytometry is a good technique for monitoring the uptake of peptides containing 4CNW.

a

P3: Ac- $\underline{-}-G R K K R R Q R R R P Q P K K K R K V-C O N H_{2}$

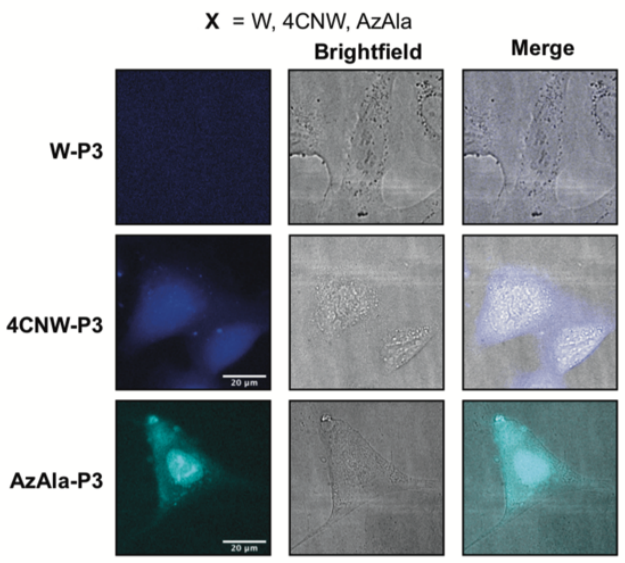

b

4CNW-P4: AC-DWLYLDDSGD-4CNW-WICG-CONH 2

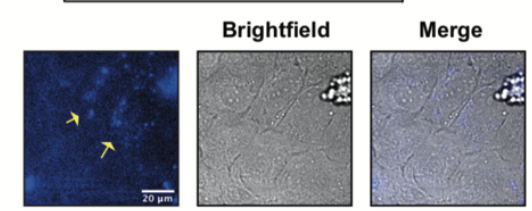

c

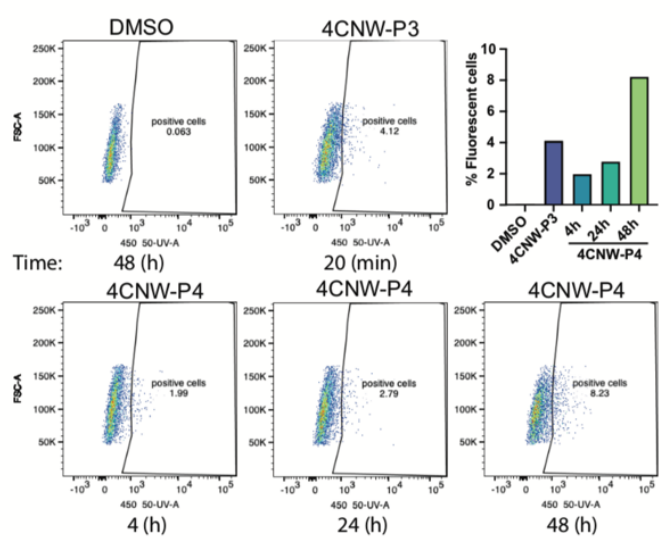

Figure 3. Uptake of $4 \mathrm{CNW} / \mathrm{AzAla}$ labelled peptides by living cells. a Live cell fluorescence microscopy images of U2OS cells after $20 \mathrm{~min}$ incubation with linear/cyclic peptides $(50 \mu \mathrm{M})$. Fluorescence of peptides is shown in blue $(4 \mathrm{CNW})$ or cyan (AzAla). Scale bar: $20 \mu \mathrm{m}$. Images were obtained by using fluorescence excitation at $340 \mathrm{~nm}$ with a ET460/50m (4CNW) or ET395/25x (AzAla) single bandpass emission filter and a Plan Fluor 60x/A1.2 WI. b Live cell fluorescence microscopy images of $\mathrm{U} 2 \mathrm{OS}$ cells after $24 \mathrm{~h}$ incubation with 
4CNW-P4 peptide $(50 \mu \mathrm{M})$. Fluorescence of peptides is shown in blue (4CNW). Scale bar: $20 \mu \mathrm{m}$. Images were obtained by using fluorescence excitation at 340 $\mathrm{nm}$ with a ET460/50m (4CNW) single bandpass emission filter and a Plan Fluor 40X/1.3NA objective. c Flow cytometry of U2OS cells incubated with DMSO, $4 \mathrm{CNW}-\mathrm{P} 3$ or $4 \mathrm{CNW}-\mathrm{P} 4$ at the indicated times. Fluorescence of a single cell suspension was acquired with a $355 \mathrm{~nm}$ laser, 450-50 detector and FACS-Diva software. Numbers indicated in graphs show percentage of positive cells.

Label-free assays are the gold standard for determining the cell permeability of cyclic peptide leads. Here, we report the incorporation of CIAc-4CNW and CIAc-AzAla into in vitro translated macrocyclic peptides via initiator reprogramming. In addition, we demonstrate $4 \mathrm{CNW}$ and AzAla are good tools for tracking peptide (linear/cyclic) penetration into cells. RaPID selections for de novo cyclic peptides initiated with $\mathrm{ClAc}-4 \mathrm{CNW}$ or CIAc-AzAla will enable the identification of peptide leads that are inherently fluorescent and can be directly assessed for cell permeability by fluorescence microscopy and flow cytometry. $4 \mathrm{CNW}$ and AzAla have also recently been used to probe protein-protein binding interactions by PET, FRET and VET. ${ }^{[9,17,18]}$ We therefore also envisage that 4CNW/AzAla initiated peptide leads could be used directly in label-free binding assays.

\section{Acknowledgements}

This work was supported by the Francis Crick Institute which receives its core funding from Cancer Research UK (FC001748), the UK Medical Research Council (FC001748), and the Wellcome Trust (FC001748). For the purpose of Open Access, the author has applied a CC BY public copyright licence to any Author Accepted Manuscript version arising from this submission. We thank Matt Renshaw from the CALM STP in the Francis Crick Institute for assistance with fluorescence microscopy. We thank the Flow Cytometry STP in the Francis Crick Institute for assistance with flow cytometry.

Keywords: RaPID • peptides • label-free • cell permeability • fluorescence imaging

[1] L. J. Walport, R. Obexer, H. Suga, Curr. Opin. Biotechnol. 2017, 48, 242-250.

[2] A. Zorzi, K. Deyle, C. Heinis, Curr. Opin. Chem. Biol. 2017, 38, 24-29.

[3] B. C. Doak, J. Zheng, D. Dobritzsch, J. Kihlberg, J. Med. Chem. 2016, 59, 2312-2327.

[4] Y. Huang, M. M. Wiedmann, H. Suga, Chem. Rev. 2019, 119, 10360-10391.

[5] J. Lee, K. Schwarz, H. Yu, A. Krüger, E. V Anslyn, A. Ellington, J. S. Moore, M. Jewett, Chem. Commun. 2021, 57, 2661-2664.

[6] D. Birch, M. V. Christensen, D. Staerk, H. Franzyk, H. M. Nielsen, Biochim. Biophys. Acta - Biomembr. 2017, 1859, 2483-2494.

[7] S. F. Hedegaard, M. S. Derbas, T. K. Lind, M. R. Kasimova, M. V. Christensen, M. H. Michaelsen, R. A. Campbell, L.
Jorgensen, H. Franzyk, M. Cárdenas, H. M. Nielsen, Sci. Rep. 2018, 8, 1-14.

[8] Y. S. Moroz, W. Binder, P. Nygren, G. A. Caputo, I. V. Korendovych, Chem. Commun. 2013, 49, 490-492.

[9] M. R. Hilaire, I. A. Ahmed, C. W. Lin, H. Jo, W. F. DeGrado, F. Gai, Proc. Natl. Acad. Sci. U. S. A. 2017, 114, 60056009.

[10] Y. Goto, T. Katoh, H. Suga, Nat. Protoc. 2011, 6, 779-790.

[11] Y. Goto, A. Ohta, Y. Sako, Y. Yamagishi, H. Murakami, H. Suga, ACS Chem. Biol. 2008, 3, 120-129.

[12] T. Kawakami, H. Murakami, H. Suga, Chem. Biol. 2008, 15, 32-42.

[13] Y. Yamagishi, I. Shoji, S. Miyagawa, T. Kawakami, T. Katoh, Y. Goto, H. Suga, Chem. Biol. 2011, 18, 1562-1570.

[14] C. E. Boville, D. K. Romney, P. J. Almhjell, M. Sieben, F. H. Arnold, J. Org. Chem. 2018, 83, 7447-7452.

[15] G. Loidl, H. J. Musiol, N. Budisa, R. Huber, S. Poirot, D. Fourmy, L. Moroder, J. Pept. Sci. 2000, 6, 139-144.

[16] M. Nawatha, J. M. Rogers, S. M. Bonn, I. Livneh, B. Lemma, S. M. Mali, G. B. Vamisetti, H. Sun, B. Bercovich, Y. Huang, A. Ciechanover, D. Fushman, H. Suga, A. Brik, Nat. Chem. 2019, 11, 644-652.

[17] T. Baumann, M. Hauf, F. Schildhauer, K. B. Eberl, P. M. Durkin, E. Deniz, J. G. Löffler, C. G. Acevedo-Rocha, J. Jaric, B. M. Martins, H. Dobbek, J. Bredenbeck, N. Budisa, Angew. Chemie - Int. Ed. 2019, 58, 2899-2903.

[18] I. A. Ahmed, J. M. Rodgers, C. Eng, T. Troxler, F. Gai, Phys. Chem. Chem. Phys. 2019, 21, 12843-12849. 


\section{Entry for the Table of Contents}

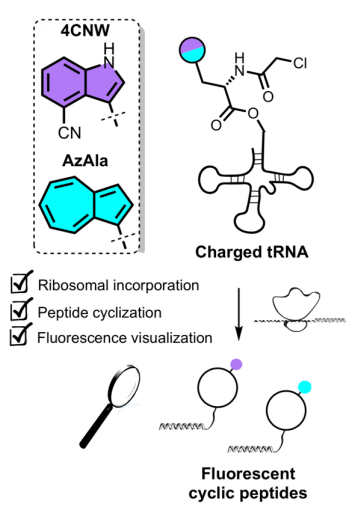

Without a trace: Fluorescent amino acids $\mathrm{AcCl}-4 \mathrm{CNW}$, AcCl-AzAla were incorporated into in vitro translated macrocyclic peptides by genetic code reprogramming. The minimally perturbing dyes were found to be good tools for tracking peptide penetration into cells by fluorescence imaging and flow cytometry.

Institute and/or researcher Twitter usernames: @Iwalport @TeresaBertran 